Western University

Scholarship@Western

Oncology Publications

Oncology Department

$1-1-2010$

\title{
A Review of Autoimmune Diseases Associated with Cancer
}

Patricia Tai

University of Saskatchewan

Edward Yu

The University of Western Ontario, edward.yu@lhsc.on.ca

Kurian Joseph

University of Alberta

Thomas Miale

Allan Blair Cancer Clinic, SK

Follow this and additional works at: https://ir.lib.uwo.ca/oncpub

Part of the Bioimaging and Biomedical Optics Commons, Oncology Commons, and the Radiology Commons

Citation of this paper:

Tai, Patricia; Yu, Edward; Joseph, Kurian; and Miale, Thomas, "A Review of Autoimmune Diseases Associated with Cancer" (2010). Oncology Publications. 70.

https://ir.lib.uwo.ca/oncpub/70 


\section{Autoimmune diseases and cancer}

Patricia Tai ${ }^{1}$ Edward Yu ${ }^{2}$, Kurian Joseph ${ }^{3}$ and Tom Miale ${ }^{4}$

${ }^{1}$ Faculty of Oncology, University of Saskatchewan, Saskatchewan, Canada; ${ }^{2}$ Department of Radiation Oncology,

University of Western Ontario, London, Canada; ${ }^{3}$ Department of Radiation Oncology, University of Edmonton,

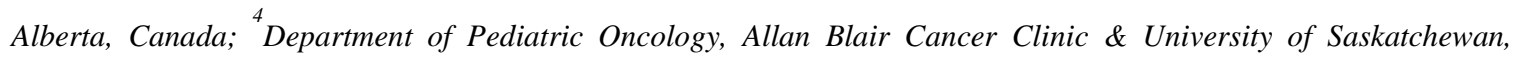
Saskatchewan, Canada

\section{TABLE OF CONTENTS}

1. Abstract

2. Introduction

3. Autoimmune disease associated with increased cancer risk

3.1. Rheumatoid arthritis

3.2. Systemic lupus erythematosus

3.3. Sjögren's syndrome

3.4. Celiac disease (Non-tropical sprue)

4. Autoimmune phenomenon with cancers - paraneoplastic syndromes

4.1. Neurological

4.1.1. Neuromuscular Junction Disorders

4.1.1.1. Lambert-Eaton myasthenic syndrome

4.1.1.2. Myasthenia Gravis

4.1.2. Dermatomyositis and polymyositis

4.2. Others

5. Perspectives - Cancer vaccines

6. References 


\section{Abstract}

This is a review of the relationship of autoimmune diseases to cancer from two perspectives. The first is to examine which autoimmune diseases are often associated with increased risk of the development of a malignancy. The second is to review which common cancers confer an increased risk of developing an autoimmune disease. In addition, some novel approaches to cancer immunotherapy are discussed.

\section{Introduction}

Emerging evidence has implicated certain environmental factors such as infections, and dietary proteins in the pathogenesis of many autoimmune diseases and cancer ${ }^{1}$. This article attempts to correlate the common factors, with recent updates from the literature. There are many unresolved observations. For example, autoimmune thyroid disease is associated with multiple myeloma [odds ratio $=5.68,95 \%$ confidence interval: $1.69-19.13]^{2}$. There is also a case report of autoimmune disease occurring in a long-term survivor with metastatic colon carcinoma treated with a novel chemo-immunotherapy regimen ${ }^{3}$. GOLFIG-1 is a new translational anticancer regimen based on the combined use of gemcitabine, oxalipatin, levofolinic acid and infusional 5-fluorouracil and subcutaneous administration of immunoadjuvant cytokines, granulocyte-macrophage-colony stimulating factor (GM-CSF) and very low dose interleukin-2 (IL-2). The patient responded to the GOLFIG regimen but developed discoid lupus erythematosus.

Primary immunodeficiencies (PIDs) are genetic disorders that predispose to frequent and severe infections, autoimmunity and cancer ${ }^{4}$. The expanded life span of such patients increases the overall risk for developing cancer, which is now estimated at 4-25\%. The type of malignancy depends on the primary immunodeficiency, the age of the patient and possibly viral infection. Non-Hodgkin lymphomas predominate, accounting for 60\% of cases. The PIDs known to be associated with increased incidence of malignancy are: common variable immunodeficiency, IgA deficiency, DNA repair disorders, as severe combined immunodeficiency, Wiskott Aldrich syndrome, Klinefelter’s syndrome, Chediak-Higashi syndrome, ataxia telangiectasia syndrome. Acquired immunodeficiency diseases include iatrogenic immunosuppression as in organ transplant recipients, acquired immunodeficiency syndrome caused by human immunodeficiency virus (HIV), and hypogammaglobulinemia. HTLV-1 is associated with adult T cell leukemia-lymphoma (ATLL). HIV produces AIDS and the resultant immune deficiency is associated with high-grade 
B-cell lymphoma. Around 60 years ago, infants were given thymic irradiation. Autoimmune diseases appear later in life. The disrupted immune system also predisposes them to cancer.

There may be many mediators and receptors involved. IL-21 is a type I cytokine ${ }^{5}$ produced by activated CD4(+) T cells, NKT cells, and Th17 cells. It has many functions: regulates immunoglobulin production, promotes B cell terminal differentiation into plasma cells, cooperatively expands CD8(+) T cells, promotes Th17 differentiation, has inhibitory effects on antigen presentation by dendritic cells, and may be pro-apoptotic for B and NK cells. Moreover, IL-21 has potent anti-tumor effects while it is also implicated in the development of autoimmune diseases. IL-21 research may potentially be helpful in the treatment of a range of illnesses.

\section{Autoimmune disease associated with increased cancer risk}

Examples of autoimmune disorders associated with development of lymphomas are rheumatoid arthritis, lupus erythematosus, Sjögren’s syndrome, Celiac disease and Hashimoto’s thyroidits.

\subsection{Rheumatoid arthritis (RA)}

Large granular lymphocyte proliferation may be present in some patients with RA and may sometimes progress to T cell large granular lymphocyte leukemia. In addition, the risk of lymphoma is increased in patients with RA, whether treated with methotrexate, or with other immunosuppressive agents or not.

\subsection{Systemic lupus erythematosus}

The risk of non-Hodgkin lymphomas appears to be increased four to five fold. In particular, angioimmunoblastic T cell lymphoma may occur more often than expected.

\subsection{Sjögren's syndrome}

Keratoconjunctivitis sicca refers to the ocular dryness associated with Sjögren's syndrome. Both Sjögren’s syndrome and keratoconjunctivitis sicca are caused by immune-mediated inflammation directed against the exocrine glands of the eye and mouth. The histologic features include extensive lymphocytic infiltration, accompanied by glandular and ductal atrophy. This lymphocytic infiltration is organized in germinal center-like structures. Salivary glands are typically enlarged.

Sjögren’s syndrome is characterized by polyclonal B cell activation as well as lymphocytic infiltration of the exocrine glands. This B cell activation predisposes some patients to the development of lymphoma. Most lymphomas 
arise from a reactive infiltrate called lymphoepithelial sialadenitis or a benign lymphoepithelial lesion. The lifetime risk of non-Hodgkin lymphoma is increased 16 to 44 times higher than expected, at approximately 5 percent.

A variety of clinical features appear to identify patients with Sjögren’s syndrome who are at increased risk for lymphoma development: cutaneous vasculitis, peripheral neuropathy, rheumatoid factor positivity, type II cryoglobulins. Risk factors identified in other studies include anti-Ro/SSA or anti-La/SSB antibody positivity and infiltration of salivary glandular tissue by dendritic cells and macrophages that express the cytokines IL-12 and IL-18.

\subsection{Celiac disease (Non-tropical sprue)}

Serologic studies, now used to confirm the diagnosis of celiac disease, include the ELISA for IgA antibodies to gliadin and the immunofluorescence test for IgA antibodies to endomysium, a structure in the smooth muscle connective tissue. Elevated endomysium IgA antibodies are almost a pathognomonic finding. The target autoantigen contained within the endomysium was identified as tissue transglutaminase. IgA-antibodies against endomysium and the endomysial autoantigen tissue transglutaminase are both highly sensitive and specific. Widespread use of these serologic tests has allowed earlier diagnosis, large scale population screening and thereby an improved understanding of its epidemiology. These patients have an increased incidence of gastrointestinal non-Hodgkin lymphomas.

\section{Autoimmune phenomenon with cancers - paraneoplastic syndromes}

Neurological diseases are defined as paraneoplastic when they occur in increased frequency in patients with cancer and are not related to a direct primary or metastatic effect of tumor, infection, metabolic abnormalities, or toxicity of therapy ${ }^{6},{ }^{7}$. Autoantibodies and evidence for cellular autoimmunity directed against neuronal, glial, or muscle cell antigens have been identified in a number of paraneoplastic neurological disorders ${ }^{8},{ }^{9}$. Over the past four decades, different investigators identified and reported these disorders using a variety of names. This review employs the nosology used by Posner (ref 7? Above): Paraneoplastic disorders are rare, but accurate diagnosis is important. For patients with an occult malignancy, correct diagnosis may lead to the early diagnosis and treatment of the underlying malignancy. Effective treatment of the neurologic disorder may improve neurologic dysfunction and improve quality of life. Equally important, proper diagnosis of a paraneoplastic disorder spares the patient an extensive and expensive search for alternative causes of the neurologic dysfunction.

\subsection{Neurological}


Examples of neurological paraneoplastic syndrome include: subacute sensory neuronopathy and encephalomyeloneuritis, limbic encephalitis, autonomic neuropathy, progressive cerebellar degeneration, paraneoplastic vision loss, opsoclonus-myoclonus, motor neuron disorders, peripheral neuropathies, neuromuscular junction disorders, muscle rigidity, dermatomyositis, polymyositis and movement disorders. Details are available in a bigger textbook (Devita). The most commonly encountered neurological paraneoplastic syndromes in the clinic are neuromuscular junction disorders, and dermatomyositis, polymyositis and will be discussed in detail as follows.

\subsubsection{Neuromuscular Junction Disorders}

\subsubsection{Lambert-Eaton myasthenic syndrome}

The autoimmune disorder of Lambert-Eaton myasthenic syndrome (LEMS) is associated with small cell lung carcinoma in $50-60 \%$ of cases. Proximal weakness is a common presenting complaint, but bulbar symptoms are uncommon. In most patients, LEMS is not a pure motor syndrome. Paresthesias are frequently reported sensory findings. The abnormality of autonomic function has been called "cholinergic dysautonomia”. Patients may report dry mouth or erectile dysfunction. Characteristic electrophysiologic abnormalities include augmentation of compound motor action potentials with repetitive stimulation. Antibodies directed against protein epitopes in the voltage-gated calcium channel of presynaptic neurons are present in most patients with LEMS. Passive transfer of antibody reproduces the characteristic electrophysiologic abnormality in animal models of LES. Immunization with a component of the P/Q-type calcium channel, synaptotagmin, produces autoantibodies and clinical disease in an animal model of LES. Unlike other paraneoplastic syndrome, most patients with LES benefit from plasmapheresis and immunosuppressive therapy. Drugs that increase presynaptic acetylcholine release may also decrease symptoms. 3,4diaminopyridine is one such agent with relatively minimal side effects. It has been postulated that patients with paraneoplastic neurological syndromes such as LEMS have an improved tumour prognosis compared to other patients with the tumour but without such neurological deficits ${ }^{10}$.

\subsubsection{Myasthenia Gravis}

Typical myasthenia gravis is associated with thymoma in approximately 15\% of cases, and autoantibodies against contractile proteins of striated muscle are associated with increased probability of underlying thymoma. All patients with myasthenia gravis should undergo thoracic imaging studies to identify thymic neoplasms. In patients with thymoma, the myasthenia gravis often remit after thymectomy. In almost all cases, the thymoma is not invasive and can be definitively treated by thymectomy. The author had come across a patient with unrecognized myasthenia gravis and died from respiratory failure. 


\subsubsection{Dermatomyositis and polymyositis}

These conditions are inflammatory myopathies characterized by the subacute development of proximal muscle weakness, with or without pain and muscle tenderness. Both conditions are usually idiopathic. Approximately $10 \%$ are associated with cancer. The myopathy usually precedes the diagnosis of the underlying cancer. Although most patients with dermatomyositis do not have cancer, patients with the disorder are probably at a higher risk for cancer. When the characteristic findings are present in men older than 40 years, there is a higher incidence of underlying cancer, and early detection should be the goal. Breast cancer is the most commonly associated cancer in women, and lung and GI cancer in men. Association with tumors of the pancreas, melanoma, germ cell tumors, nasopharyngeal carcinoma, and lymphoma has also been reported.

In addition to proximal symmetric muscle weakness and skin changes, there is usually an elevation in muscle creatinine kinase. Electromyography is consistent with a myopathic process. A muscle biopsy usually shows inflammatory infiltrates, necrotic fibres, and atrophic fibres, sometimes in a perifascicular pattern. An immunemediated intramuscular angiopathy leads to eventual ischemia and muscle fiber necrosis. IgG, IgM, and complement deposits are found in small blood vessels. Cellular inflammatory infiltrates include B cells, macrophages, and CD4+ T cells.

Immunosuppression has not been tested specifically in patients with myositis. It is unclear if antineoplastic therapy leads to improvement in the muscle disease in the absence of concomitant immunosuppression. Both dermatomyositis and polymyositis syndromes follows an inconsistent course and often independent of the cancer outcome.

\subsection{Others}

Autoimmune hemolytic anemias are typically associated with B-cell malignancies, including chronic lymphocytic leukemia and lymphoma, and arise secondary to immunoregulatory abnormalities in these diseases, rather than to a direct secretion of tumor-derived substances ${ }^{11}$. Hallmarks of this disorder disease are a positive direct antiglobulin test (Coombs’) result, elevated reticulocyte count, decreased haptoglobin level, and elevated lactate dehydrogenase level. Warm or cold antibody immune hemolytic anemias may be seen particularly in the small 
lymphocytic type. Additional autoimmune phenomena, such as circulating anticoagulants (e.g., acquired von Willebrand's disease) may occur, especially in the small lymphocytic lymphomas.

Warm antibody hemolytic anemia is most commonly associated with lymphoma, chronic lymphocytic leukemia, and mucin-producing adenocarcinoma. Cold agglutinin disease is most common in Waldenström's macroglobulinemia and lymphomas ${ }^{12}$. Autoimmune hemolytic anemia is rarely associated with solid tumors. However, an association with ovarian, GI, lung, breast, and renal cell cancers has been reported also[Akard AGAIN]. Corticosteroid treatment appears to be less effective in autoimmune hemolytic anemia associated with carcinomas than in those that are idiopathic or associated with lymphoid malignancies. The Coombs' test result may revert to negative with control of the tumor.

\section{Perspectives - Cancer vaccines}

Immunotherapy for melanoma has undergone significant change since the first attempts to treat patients with high dose IL-2. There are now strategies to boost patient antitumor immunity through vaccination, treatment with agents that augment host immunity, and adoptive cell transfer ${ }^{13}$. The first two strategies have yielded only limited clinical success, but adoptive cell transfer therapy, particularly following a lymphodepleting, preconditioning regimen has resulted in objective response rates approaching $50 \%$.

Over the last century, vaccine studies have demonstrated that the human immune system, with appropriate help, can limit or prevent infection against otherwise lethal pathogens ${ }^{14}$. Encouraged by these results, success in animal models and numerous well-documented reports of immune-mediated melanoma regression in humans, investigators developed melanoma vaccines. However, despite considerable laboratory evidence for vaccine-induced immune responses, clinical responses remain poor. Recent studies have elucidated several mechanisms that hinder or prevent the creation of successful vaccines and suggest novel approaches to overcome these barriers. Unraveling the mechanisms of autoimmunity, dendritic cell activation, regulatory $\mathrm{T}$ cells and Toll-like receptors will generate novel vaccines that, when used in conjunction with standard adjuvant therapies, may result in improved clinical outcomes.

Balancing antitumor efficacy, autoimmunity, and reconstitution of a functioning immune system remain challenging and potentially life-threatening issues. Nonmutated tissue differentiation antigens expressed by tumors are attractive targets for cancer immunotherapy, but the consequences of a highly effective antitumor immune response on 
self-tissue have not been fully characterized. The infusion of ex vivo expanded adoptively transferred melanoma/melanocyte-specific CD8+ T cells that mediated robust tumor killing also induced autoimmune destruction of melanocytes in the eye ${ }^{15}$. This severe autoimmunity was associated with the up-regulation of MHC class I molecules in the eye and high levels of IFN-gamma derived from both adoptively transferred CD8+ T cells and host cells. Furthermore, ocular autoimmunity required the presence of the IFN-gamma receptor on target tissues. Data compiled from >200 eyes and tumors in 10 independently performed experiments revealed a highly significant correlation $(\mathrm{P}<0.0001)$ between the efficacy of tumor immunotherapy and the severity of ocular autoimmunity. Administration of high doses of steroids locally mitigated ocular autoimmunity without impairing the antitumor effect. These findings have particular importance for immunotherapies directed against self-antigens and highlight the need for targeting unique tumor antigens not expressed in critical tissues.

\section{References}

\footnotetext{
${ }^{1}$ Vojdani A. Antibodies as predictors of complex autoimmune diseases. Int J Immunopathol Pharmacol. 2008 Apr-Jun;21(2):267-78.

${ }^{2}$ Dalamaga M, Karmaniolas K, Papadavid E, Pelecanos N, Migdalis I. Association of thyroid disease and thyroid autoimmunity with multiple myeloma risk: a case-control study. Leuk Lymphoma. 2008 Aug;49(8):1545-52.

${ }^{3}$ Correale P, Fioravanti A, Bertoldi I, Montagnani F, Miracco C, Francini G. Occurrence of autoimmunity in a long-term survivor with metastatic colon carcinoma treated with a new chemo-immunotherapy regimen. J Chemother. 2008 Apr;20(2):278-81.

${ }^{4}$ Salavoura K, Kolialexi A, Tsangaris G, Mavrou A. Development of cancer in patients with primary immunodeficiencies. Anticancer Res. 2008 Mar-Apr;28(2B):1263-9.
}

${ }^{5}$ Spolski R, Leonard WJ. The Yin and Yang of interleukin-21 in allergy, autoimmunity and cancer. Curr Opin Immunol. 2008 Jun;20(3):295-301. Epub 2008 Jun 12.

${ }^{6}$ Clouston, PD, De Angelis LM, Posner JB. The spectrum of neurologic disease in patients with systemic cancer. Ann Neurol 1992;31:268.

${ }^{7}$ Paraneoplastic syndromes. In: Posner JB, ed. Neurologic complications of cancer. Philadelphia: FA Davis,1995:353.

${ }^{8}$ Dalmau J, Posner JB. Neurologic paraneoplastic antibodies (anti-Yo, anti-Hu, anti-Ri): the case for a nomenclature based on antibody and antigen specificity. Neurology 1994;44:2241.

${ }^{9}$ Posner JB, Furneaux HM. Paraneoplastic syndromes. In: Waksman BH, ed. Immunologic mechanisms in neurologic and psychiatric disease. New York: Raven Press, 1990:187.

${ }^{10}$ Maddison P, Lang B. Paraneoplastic neurological autoimmunity and survival in small-cell lung cancer. J Neuroimmunol. 2008 Sep 15;201-202:159-62. 
${ }^{11}$ S.M. Arnold, F.S. Lieberman, K.A. Foon. Chap 45. V.T. DeVita, Jr. S. Hellman, S.A. Rosenber. Editors. Cancer Principles and practice of Oncology. Philadelphia: Lippincott Williams \& Wilkins, 2005.

${ }^{12}$ L.P. Akard, J. Brandt, L. Lee L, et al. Chronic T cell lymphoproliferative disorder and pure red cell aplasia. Am J Med 83, 1069 (1987).

${ }^{13}$ Fang L, Lonsdorf AS, Hwang ST. Immunotherapy for advanced melanoma. J Invest Dermatol. 2008 Nov;128(11):2596-605.

${ }^{14}$ Riley LB, Agarwala SS. Melanoma vaccines. Expert Rev Vaccines. 2008 Sep;7(7):937-49.

${ }^{15}$ Palmer DC, Chan CC, Gattinoni L, Wrzesinski C, Paulos CM, Hinrichs CS, Powell DJ Jr, Klebanoff CA, Finkelstein SE, Fariss RN, Yu Z, Nussenblatt RB, Rosenberg SA, Restifo NP. Effective tumor treatment targeting a melanoma/melanocyte-associated antigen triggers severe ocular autoimmunity. Proc Natl Acad Sci U S A. 2008 Jun 10;105(23):8061-6. 